\title{
Successful Evaluation of Spinal Mobility Measurements With the Epionics SPINE Device in Patients With Axial Spondyloarthritis Compared to Controls
}

\author{
David Kiefer ${ }^{1}\left(\mathbb{D}\right.$, Xenofon Baraliakos $^{1}$, Daniela Adolf ${ }^{2}$, Varvara Chatzistefanidi ${ }^{1}$, Ilka Schwarze ${ }^{3}$, \\ Uwe Lange $^{4}$, Jan Brandt-Jürgens ${ }^{5}$, Edgar Stemmler ${ }^{6}$, Sabine Sartingen ${ }^{6}$, and Jürgen Braun ${ }^{1}$
}

\begin{abstract}
Objective. Epionics SPINE (ES), a novel device that measures spinal movements using electronic sensors including range of motion (RoM) and speed (range of kinematics [RoK]), has already been validated in patients with mechanical back pain and healthy individuals. This study aimed to evaluate ES for quantification of spinal mobility in patients with axial spondyloarthritis (axSpA).

Methods. A total of 153 individuals, 39 female and 114 male, were examined including 134 patients with axSpA, of whom 40 had nonradiographic (nr)-axSpA, 94 had radiographic (r)-axSpA; 19 were healthy controls (HCs). The results were compared using mean ES scores and modeling was performed using multivariable logistic regression models resulting in good validity and high discriminative power.

Results. ES measurements showed meaningful differences between patients with axSpA and HCs (all $P<0.001)$, as well as between $r-$ and nr-axSpA $(P<0.01)$. In patients with axSpA, a negative correlation between ES and Bath Ankylosing Spondylitis Metrology Index values was found: $-0.76 \leq r \leq-0.52$ $(P<0.05)$. Bath Ankylosing Spondylitis Functional Index scores showed a similar trend $(r>-0.39)$. Patients with $\mathrm{r}$-axSpA had a more limited and slower spinal mobility than those with nr-axSpA. Other patient-reported outcomes almost did not correlate.

Conclusion. This study shows that the ES is an objective performance measure and a valid tool to assess spinal mobility in axSpA, also based on the Outcomes Measures in Rheumatology (OMERACT) criteria. RoK and RoM scores provide additional information on physical function of patients with axSpA.
\end{abstract}

Key Indexing Terms: axial spondyloarthritis, BASMI, spinal measurements, spinal mobility

Axial spondyloarthritis (axSpA) is a chronic rheumatic disease that is characterized by inflammatory back pain and several other articular and extraarticular disease manifestations as well as substantial comorbidity. ${ }^{1}$ Based on the 2009 Assessment of

AbbVie sponsored this study and contributed to the design, study conduct, and analysis. AbbVie participated in the interpretation of data, review, and approval of the manuscript.

${ }^{I} D$. Kiefer, $M D, X$. Baraliakos, MD, V. Chatzistefanidi, MD, J. Braun, $M D$, Rheumazentrum Ruhrgebiet, Ruhr-University Bochum, Bochum;

${ }^{2} D$. Adolf, PhD, StatConsult, Gesellschaft für klinische und

Versorgungsforschung mbH, Magdeburg; ${ }^{3}$ I. Schwarze, MD, Praxis für Rheumatologie, Leipzig; ${ }^{4}$ U. Lange, MD, Rheumatologie und klinische Immunologie Campus Kerckhoff, Universität Gießen, Bad Nauheim; ${ }^{5}$ J. Brandt-Jürgens, MD, Rheumatologische Schwerpunktpraxis, Berlin; ${ }^{6}$ E. Stemmler, PhD, S. Sartingen, PhD, Medical Immunology, AbbVie Deutschland GmbH \& Co. KG, Wiesbaden, Germany.

$D K, X B, D A, V C, I S, U L, J B J$, and $J B$ have received speaker or consulting fees from AbbVie. ES and SS are employees of AbbVie and may own AbbVie stock.

Address correspondence to Dr. D. Kiefer, Rheumazentrum Rubrgebiet Herne Rubr-University Bochum, Claudiusstrasse 45, 44649 Herne, Germany.

Email:david.kiefer@elisabethgruppe.de.

Accepted for publication August 3, 2021.
Spondyloarthritis international Society (ASAS) classification criteria, the classical ankylosing spondylitis (AS) or radiographic axSpA ( $\mathrm{r}-\mathrm{axSpA})$ and nonradiographic axSpA (nr-axSpA) have been differentiated according to the presence or absence of definite radiographic changes in the sacroiliac joints. ${ }^{2}$ Importantly, back pain in patients with axSpA may well have reasons other than axial inflammation or new bone formation. ${ }^{3}$ The course of axSpA is rather different, and the most important outcome variables assess disease activity, function, mobility, and spinal structural changes. Commonly used measures are the Bath Ankylosing Spondylitis Disease Activity Index (BASDAI), Ankylosing Spondylitis Disease Activity Score (ASDAS), Bath Ankylosing Spondylitis Functional Index (BASFI), Bath Ankylosing Spondylitis Metrology Index (BASMI), the Bath Ankylosing Spondylitis Global Score (BAS-G), the Ankylosing Spondylitis Quality of Life score (ASQoL), and the ASAS Health Index (ASAS HI).45,6,7,8,9 Previously, the AS Performance Index (ASPI) has been introduced as a first performance measure. ${ }^{10}$

Both axial inflammation (as assessed by magnetic resonance imaging) and structural damage, mainly as new bone formation (commonly assessed by conventional radiography and the modified Stoke Ankylosing Spondylitis Spine Score ${ }^{11}$ ), influence the function and mobility of patients with AS. ${ }^{12}$ The most 
frequently performed mobility measures in axSpA quantify anterior and lateral spinal flexion, cervical rotation, occiput-to-wall distance, and chest expansion. ${ }^{6}$ The first 4 are part of the BASMI which, in addition, looks at the range of motion (RoM) of the hips. Among different methods to calculate BASMI, the linear BASMI method was most sensitive to change. ${ }^{10}$ The tests for bending, putting on socks, and getting up from the floor were previously included in the ASPI because of their high standardized response mean in patients with AS treated with a tumor necrosis factor inhibitor. ${ }^{13}$

The Epionics SPINE (ES) device, a noninvasive tool to assess spinal mobility of the spine that has been evaluated in patients with mechanical back pain, ${ }^{14,15,16}$ has recently been tested in a small pilot study in patients with axSpA. ${ }^{17}$ The system enables the assessment of back movements using strain gauge sensors located along flexible circuit board strips placed at predefined areas of the back to provide a sensitive measure of electrical resistance, and thus the aperture angles, according to the curvature in each of six 50-mm sensor segments. The accuracy of the system was proven in healthy subjects with an excellent intraclass correlation coefficient (ICC) average $>0.98$ and a very good test-retest repeatability with ICCs $>0.98 .^{18}$

In addition to the exact assessment of spinal mobility in angular degrees (RoM), the speed of the executed movement can also be digitally recorded as the range of kinematics (RoK). The ES is able to identify any movement of the lumbar spine and may also be used continuously for up to 24 hours. Immediately after the examination, a spider diagram is automatically created to show patients' scores. Traditional tools to assess function and mobility do not measure spinal RoM and RoK in all planes with good accuracy; these also have limited reliability and sensitivity to change on the individual patient level and in longitudinal assessments but are more informative at a group level. ${ }^{19,20,21,22}$ Therefore, it was a logical step to evaluate the ES also in patients with axSpA. Thus, a national, multicenter study was designed to investigate the different measurement tools to assess function and spinal mobility in patients with nr-axSpA and $\mathrm{r}$-axSpA in comparison to healthy controls (HCs) according to the Outcome Measures in Rheumatology (OMERACT) criteria. ${ }^{23}$ We chose to apply the specific imaging aspects of the OMERACT criteria to our investigation as there are currently no OMERACT criteria for computerized metric measurements.

\section{METHODS}

This study was designed as a national, cross-sectional multicenter trial in which consecutive patients aged $\geq 18$ years diagnosed with axSpA were prospectively included after written informed consent was obtained. Patients who had undergone spinal surgery and pregnant women were excluded. Healthy individuals without back pain served as HCs. Patients with axSpA were subdivided into 2 subgroups based on the 2009 ASAS criteria and the 1984 modified New York criteria as r-axSpA and nr-axSpA. In the nomenclature, no difference was made between the terms $r$-ax $S p A$ and $A S$. Five German centers participated. In order to avoid diurnal variability, all investigations were carried out in the morning, before noon.

Further, the results of the ES measurements of patients with axSpA were compared with HCs who had been recruited for this study; a historical cohort of HCs was added to be able to better compare limitations of spinal movement related to age..$^{14,24}$
For this study, required ethics approval has been obtained from the independent ethics committee of the Medical Association of Westphalia-Lippe and the University of Münster on January 20, 2015 (reference number 2014-277-f-S, study code: 10234). This study fully complies with the Declaration of Helsinki (Fortaleza/BR, 2013), International Council for Harmonisation of Technical Requirements for Pharmaceuticals in Human Use guidelines of Good Clinical Practice, Guidelines of Good Epidemiology Practice, and the respective applicable supranational, international, national, and local regulations and norms. Written informed consent has been obtained from the subjects (or their legally authorized representative). With regard to the clinical trial registration, the presented study is a noninterventional, observational clinical study with a licensed medical device as defined by the International Committee of Medical Journal Editors criteria.

Assessment tools. All participants underwent all physical examinations with the ES and the BASMI. In addition, patients were asked to complete the following questionnaires: BASDAI, BASFI, BAS-G, ASAS HI, ASDAS and the 12-item Short Form Health Survey with both the physical component summary (PCS) and the mental component summary (MCS) included.

Since these assessment tools were developed for patients with axSpA, HCs were not asked to complete them. Thus, correlation analyses could only be performed in patients with axSpA.

Measurements with the ES. The ES is a noninvasive electronic class IIa-certified movement analysis system distributed by the MCG motion capture $\mathrm{GmbH}$ and consists of a sensor strip, memory unit, and docking station (Supplementary Figure 1, available with the online version of this article). To compare this device with current measurements, a choreography of movements was developed (Supplementary Figure 2). Before starting the measurement, each participant's demographic data including age and sex, as well as data from the physical examination such as height, weight, and the distance between C7 and S1 (C7-posterior superior iliac spine [PSIS]) were entered into the ES software. The sensors were placed bilaterally at a distance of $5 \mathrm{~cm}$ along the spine. The standardized paravertebral position of the sensors allows it to record movements and rotations outside of the sagittal plane. The vertical plane is defined by the positioning of the caudal segment at the level of the PSIS.

After the signal "go," patients had to perform predefined exercises (choreography) to assess and record their spinal mobility. All exercises had to be performed 3 times in a row and as fast as possible. The time between the exercise was measured as interval time.

The ES measurement variables used in this study included flexion, extension, lateral flexion, rotation, and the pile test. The pile test is a complex performance combining different movements including flexion, extension, and rotation (to lift a virtual object next to your right or left foot over the opposite side of the patient's head). The sensors of the ES calculated the RoM, measured in angular degrees. The maximum speed with which the exercises were performed (RoK) was measured in angular degrees/second.

Objectives and statistical methods. The main aim of this study was to demonstrate that ES measurements can differentiate between health and disease. Therefore, the spinal mobility of patients with axSpA was assessed using ES and BASMI scores and the results of various questionnaires, and the scores were compared to those of HCs. Further, the specific imaging aspects of the OMERACT criteria of truth, discrimination, and feasibility were adapted and used for validation. In addition, the ES variables of patients with axSpA and another $\mathrm{HC}$ cohort based on aggregated historical data were compared by $t$ tests. ${ }^{14,24}$

The scores of spinal measurements (ES and BASMI) and the questionnaires were directly compared between axSpA (both r-axSpA and nr-axSpA) and HCs. The correlation between ES scores and questionnaires was calculated using Spearman correlation coefficient.

For the analyses of ES variables to differentiate between the groups, analysis of covariance (ANCOVA) was performed including adjustments for sex, age, and BMI. The corresponding differences in standardized questionnaires were assessed using $t$ tests, since they had already been adjusted 
for sex and age. The results are summarized in the form of heatmaps of the individual ES variables RoM and RoK. Effect sizes are visualized by different colors (Figure 1; Supplementary Figure 3 and 4, available with the online version of this article). Further, to discriminate between patients and controls, as well as between axSpA subgroups, receiver-operating characteristic curve (ROC) analyses based on single variables and logistic regression models of covariable-adjusted ES variables were performed.

In order to estimate a comprehensive logistic regression model, derived and raw ES variables were chosen. This was realized using a predefined procedure for variable selection and combination: on one hand, a factor analysis (varimax rotation, eigenvalues $\geq 1$ ) was performed to detect the most relevant and largely uncorrelated raw ES variables; on the other hand, a logistic regression with all derived RoM and RoK variables was performed to assess the most relevant ES variable. The resulting variables were chosen to estimate the comprehensive model consisting of covariables (sex, age, and $\mathrm{BMI}$ ), the most relevant $\mathrm{ES}$ variable, as well as supporting raw $\mathrm{ES}$ variables. Finally, the comprehensive model (to discriminate between axSpA subgroups only) was further enlarged by the most relevant questionnaire. Only complete cases were used and ES variables with $>10 \%$ missing were not included. The results were compared using a fixed minimal specificity of $\geq 80 \%$ through estimates for sensitivity and area under the curve (AUC), as well as the McNemar exact test to test systematic improvements by a model. All analyses were performed with the software SAS 9.4 (SAS Institute) and deliberately reviewed to the full level of significance of $5 \%$.

\section{RESULTS}

Demographics and baseline. A total of 153 individuals were prospectively included in the study. Of these, 134 were patients with axSpA ( 40 with nr-axSpA and 94 with r-axSpA or AS) and 19 were HCs. Thirty-nine females and 114 males underwent all examinations. Patients with r-axSpA (mean age $47.6 \pm 11.7 \mathrm{yrs}$ ) were older than patients with nr-axSpA $(38.9 \pm 11.9 \mathrm{yrs})$ and the HCs $(36.7 \pm 11.9$ yrs). BMI was comparable between groups (Supplementary Table 1, available with the online version of this article). The mean disease duration in patients with $\mathrm{r}$-axSpA was $10.6 \pm 10.1$ years, and the first onset of symptoms (mean) was prior to $19.0 \pm 11.5$ years compared to $3.9 \pm 6.9$ and $9.9 \pm 9.9$ years (mean) of the patients with nr-axSpA, respectively (Supplementary Table 1).

An HLA-B27 allele was detected in 95 of 134 patients with axSpA (70.9\%) and in 15 cases, the HLA-B27 status was not known.

Results of the questionnaires and BASMI in r-axSpA and $n r$-axSpA. Disease activity scores (BASDAI and ASDAS) were comparable in patients with $r$-axSpA and nr-axSpA. Physical function (BASFI) and spinal mobility (BASMI) were worse in patients with $r$-axSpA than in patients with nr-axSpA. BAS-G and ASAS HI showed similar results in these groups, whereas PCS scores of the 36-item Short Form Health Survey were lower in patients with r-axSpA than in patients with nr-axSpA, whereas the MCS scores were rather equal (Supplementary Table 1, available with the online version of this article).

Correlation between ES, BASMI, and patient-reported outcomes. The correlation between ES scores, assessments of spinal mobility, and standardized questionnaires of patients with axSpA is shown in Figure 1.

BASMI scores showed a significant negative correlation with all ES scores $(-0.76 \leq r \leq-0.52)$. Within the individual measurements of the BASMI, the lateral lumbar flexion, lumbar flexion, cervical rotation, and the tragus-to-wall distance showed the strongest correlation with RoM and RoK, whereas a weaker correlation was found for the intermalleolar distance (Supplementary Figure 3, available with the online version of this article).

The ES scores showed only a weak negative correlation with the BASFI $(r>-0.39)$ and almost no significant correlation with other patient-reported outcomes (Figure 1).

Discrimination between axSpA and HC with the ES. The descriptive statistics of ES of patients and HCs are shown in Supplementary Table 2 (available with the online version of this article). In the absence of questionnaire data for HCs, all analyses looking at discrimination between patients with axSpA and HCs focused on individual ES scores, on combinations of the covariables sex, age, and BMI, and on selected ES variables based on factor analyses.

In comparison with the historical HCs, patients with axSpA showed a highly significant difference in RoK flexion and extension in all age groups (Supplementary Figure 5, available with the online version of this article).

The unadjusted differences between axSpA and HCs for ES scores for RoK and RoM show significant results (all $P \leq 0.001$; Supplementary Table 3, available with the online version of this article). Corresponding ANCOVA results including covariable adjustments for sex, age, and BMI confirmed the significant discrimination between patients with axSpA and HCs (Table 1). This is also visualized in the second column of the heatmap (Supplementary Figure 4).

In addition, the differences in ES means between axSpA and HCs and discriminating ability of covariable-adjusted ES variables were assessed. To make sure those results were not based mainly on the discriminative power of covariables (sex, age, or $\mathrm{BMI}$ ), ROC analyses were repeated for the covariables only and the ES variable only. Finally, they were compared with the covariable-adjusted models; an example is given in Figure 2 for "range of kinematics in rotation."

For the majority of the ES results, the RoK and RoM were useful to distinguish between SpA and HCs, in addition to the demographic variables. Further, the ROC of the comprehensive model consisting of covariables (sex, age, and BMI), the most relevant ES variables, as well as supporting raw ES variables (details given in Table 2 and Supplementary Table 4, available with the online version of this article) were compared to the covariable-adjusted model of the most relevant $\mathrm{ES}$ variable for a fixed specificity of a minimum $\geq 80 \%$. The corresponding AUC curves are given in Figure 2. The comprehensive model reached a significantly higher sensitivity $(P=0.007)$. The corresponding results are shown in the last column of Table 2. This means that including additional raw ES variables significantly improves sensitivity. For the fixed specificity of approximately $80 \%$-in this case 16 of 19 HCs (84.2\%) - the comprehensive model (Table 2) was able to identify 118 of 127 patients in the axSpA group as true positives ( $92.9 \%$ sensitivity). In comparison with the BASMI (73.9\% sensitivity), the covariable-adjusted model (Table 2) using RoK rotation ( $85.9 \%$ sensitivity), as well as the comprehensive model ( $92.9 \%$ sensitivity) yielded a higher sensitivity $(P=0.008$ and $P<0.0001$, respectively). 


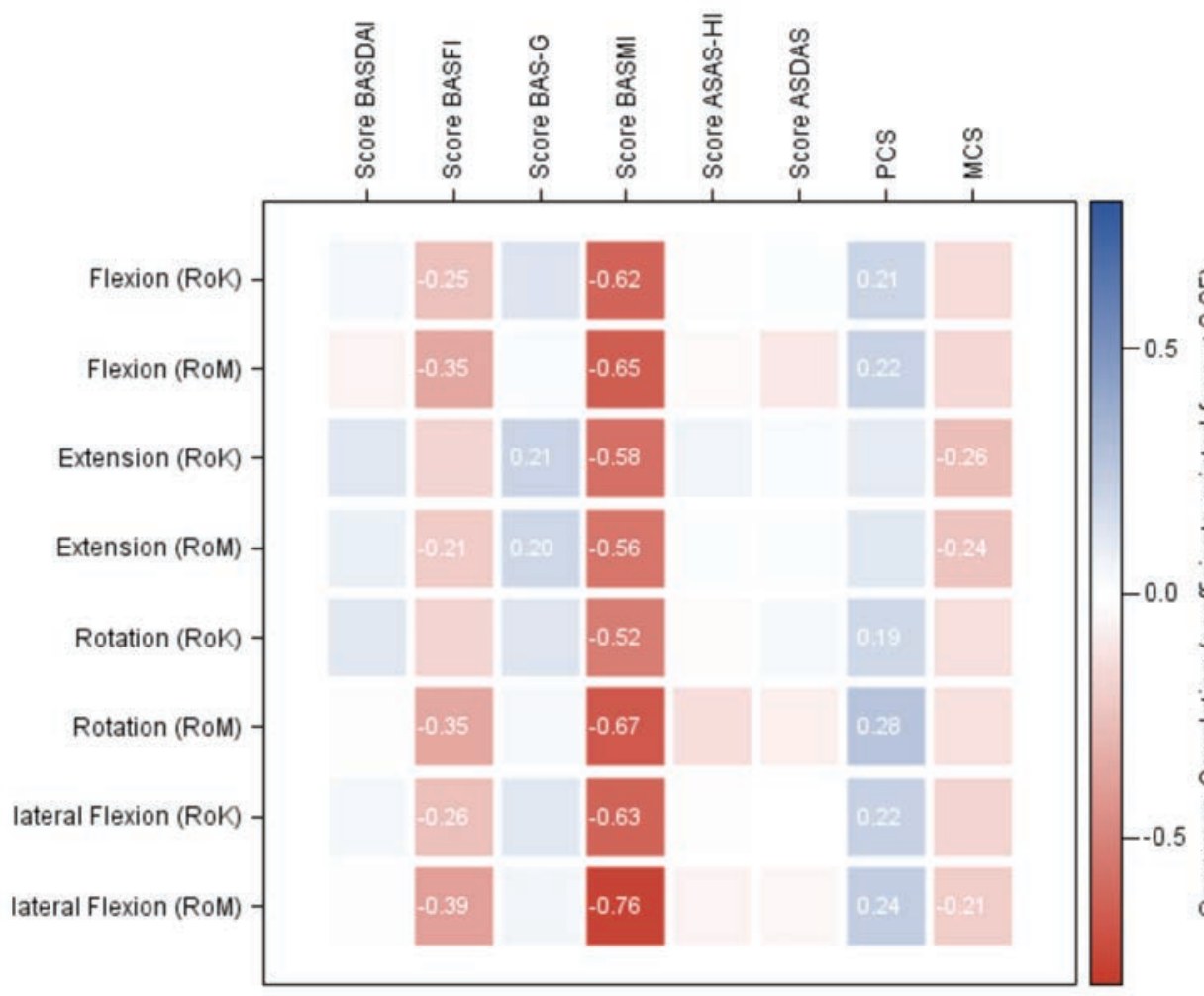

Figure 1. Heatmap of results of Spearman correlation analyses between Epionics SPINE variables and standardized questionnaires and mobility measurements for patients with axial spondyloarthritis. Red indicates a negative effect size, blue indicates a positive one, and white indicates

no effect. Significant effects or correlations $\checkmark$ are additionally displayed. Correlation a coefficient $(r)$ values are only depicted in 을 case of significance. ASDAS: Ankylosing Spondylitis Disease Activity Score; AS-HI: Ankylosing Spondylitis Health Index;

BASDAI: Bath Ankylosing Spondylitis

Disease Activity Score; BASFI: Bath

Ankylosing Spondylitis Functional Index;

BAS-G: Bath Ankylosing Spondylitis

Global Score; BASMI: Bath Ankylosing

을 Spondylitis Metrology Index; MCS:

Mental Component Summary of Medical

¿ Outcomes Study 12-item Short Form

Survey; PCS: Physical Component

Summary of Medical Outcomes Study

12-item Short Form Survey; RoK: range of

के kinematics: RoM: range of motion.

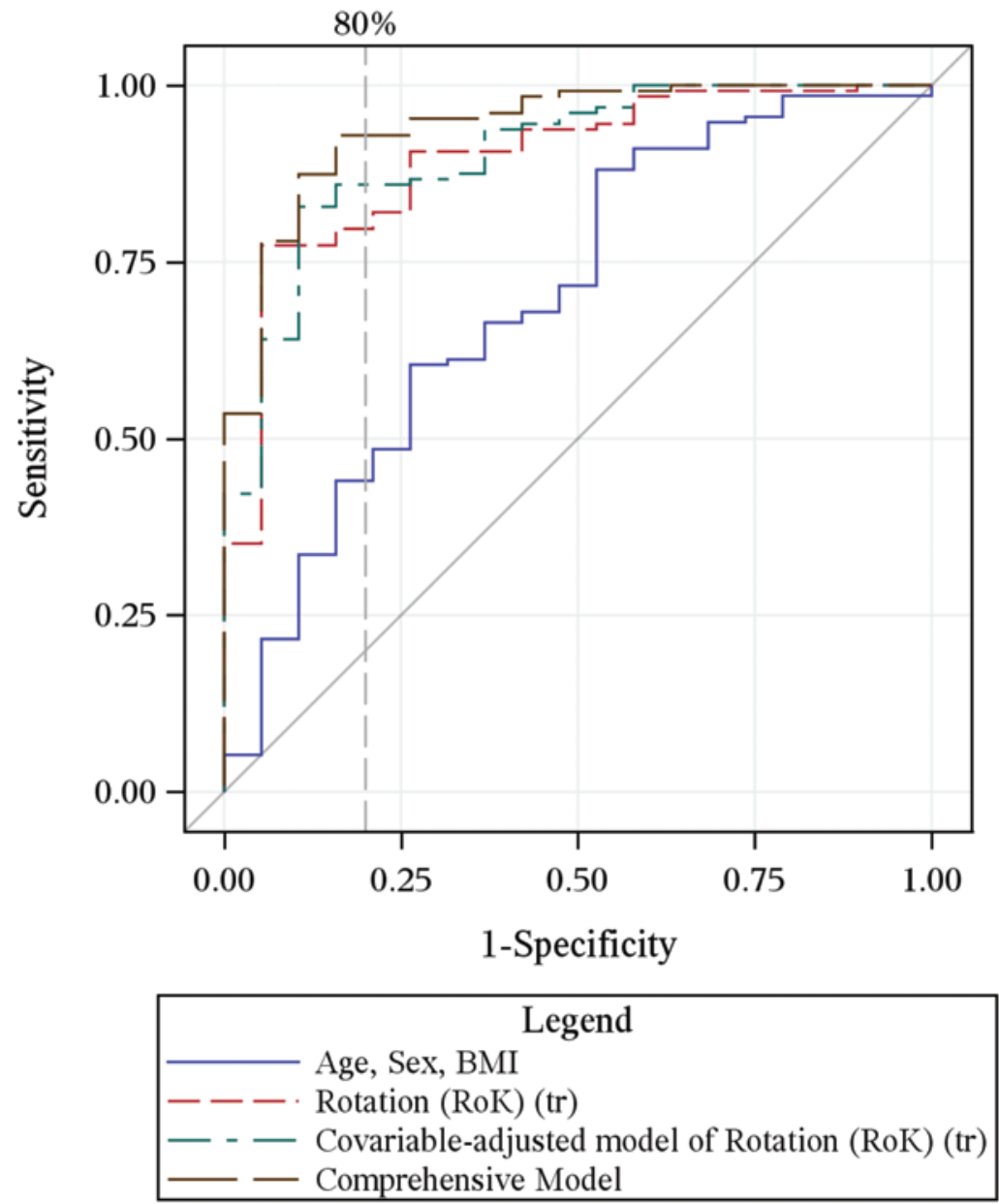

Figure 2. ROC curves for modeling axSpA of the single Epionics SPINE variable RoK in rotation; covariables only, as well as the covariable-adjusted model of this Epionics SPINE variable and the comprehensive model (missing data are excluded casewise). axSpA: axial spondyloarthritis; ROC: receiver-operating characteristic curve; RoK: range of kinematics; tr: log-transformed. 
Table 1. ANCOVA results of ES and covariable-adjustments for age, sex, and BMI between patients with axSpA and HCs.

\begin{tabular}{|c|c|c|c|c|}
\hline Dependent & Independent & Sum of Squares & $F$ Statistic & $\operatorname{Pr}>F$ \\
\hline \multirow[t]{4}{*}{ Flexion (RoK) (tr) } & Diagnosis & 73.61 & 21.94 & $<0.001$ \\
\hline & Age & 106.56 & 31.76 & $<0.001$ \\
\hline & Sex & 91.86 & 27.38 & $<0.001$ \\
\hline & BMI & 0.68 & 0.20 & 0.65 \\
\hline \multirow[t]{4}{*}{ Flexion (RoM) } & Diagnosis & 1857.06 & 12.44 & $<0.001$ \\
\hline & Age & 5192.34 & 34.79 & $<0.001$ \\
\hline & Sex & 4935.91 & 33.08 & $<0.001$ \\
\hline & BMI & 480.63 & 3.22 & 0.08 \\
\hline \multirow[t]{4}{*}{ Extension (RoK) (tr) } & Diagnosis & 62.06 & 22.03 & $<0.001$ \\
\hline & Age & 72.94 & 25.89 & $<0.001$ \\
\hline & Sex & 35.75 & 12.69 & $<0.001$ \\
\hline & BMI & 2.16 & 0.77 & 0.38 \\
\hline \multirow[t]{4}{*}{ Extension (RoM) } & Diagnosis & 1060.44 & 11.12 & 0.001 \\
\hline & Age & 1958.84 & 20.55 & $<0.001$ \\
\hline & Sex & 932.33 & 9.78 & 0.002 \\
\hline & BMI & 0.02 & 0.00 & 0.99 \\
\hline \multirow[t]{4}{*}{ Rotation (RoK) (tr) } & Diagnosis & 498.72 & 50.39 & $<0.001$ \\
\hline & Age & 99.32 & 10.03 & 0.002 \\
\hline & Sex & 74.53 & 7.53 & 0.007 \\
\hline & BMI & 1.82 & 0.18 & 0.67 \\
\hline \multirow[t]{4}{*}{ Rotation (RoM) } & Diagnosis & 7189.55 & 28.25 & $<0.001$ \\
\hline & Age & 4918.80 & 19.33 & $<0.001$ \\
\hline & Sex & 4444.87 & 17.46 & $<0.001$ \\
\hline & BMI & 259.71 & 1.02 & 0.31 \\
\hline \multirow[t]{4}{*}{ Lateral flexion (RoK) (tr) } & Diagnosis & 308.68 & 32.87 & $<0.001$ \\
\hline & Age & 175.32 & 18.67 & $<0.001$ \\
\hline & Sex & 99.14 & 10.56 & 0.001 \\
\hline & BMI & 0.55 & 0.06 & 0.81 \\
\hline \multirow[t]{4}{*}{ Lateral flexion (RoM) } & Diagnosis & 5357.25 & 25.80 & $<0.001$ \\
\hline & Age & 7699.94 & 37.08 & $<0.001$ \\
\hline & Sex & 6292.44 & 30.30 & $<0.001$ \\
\hline & BMI & 98.10 & 0.47 & 0.49 \\
\hline
\end{tabular}

Statistically significant values are indicated in bold. ANCOVA: analysis of covariance; axSpA: axial spondyloarthritis; ES: Epionics SPINE; HC: healthy control; RoK: range of kinematics; RoM: range of motion; tr: log-transformed.

Table 2. ROC curves for modeling axSpA of covariable-adjusted models of single derived ES variables, the comprehensive model, and BASMI.

\begin{tabular}{|c|c|c|c|c|c|}
\hline & AUC & LCL & UCL & Spec $\geq 80 \%(\mathrm{n} / \mathrm{N})$ & Sens $(n / N)$ \\
\hline Rotation (RoK) & 0.903 & 0.832 & 0.975 & $84.2(16 / 19)$ & $85.9(110 / 128)$ \\
\hline Lateral flexion (RoK) & 0.881 & 0.806 & 0.955 & $84.2(16 / 19)$ & $83.6(107 / 128)$ \\
\hline Flexion (RoK) & 0.872 & 0.801 & 0.944 & $84.2(16 / 19)$ & $73.9(99 / 134)$ \\
\hline Lateral flexion (RoM) & 0.858 & 0.776 & 0.940 & $84.2(16 / 19)$ & $69.5(89 / 128)$ \\
\hline Extension (RoK) & 0.830 & 0.730 & 0.930 & $84.2(16 / 19)$ & $74.8(98 / 131)$ \\
\hline Comprehensive model & 0.941 & 0.887 & 0.995 & $84.2(16 / 19)$ & $92.9(118 / 127)$ \\
\hline BASMI & 0.880 & 0.821 & 0.938 & $89.5(17 / 19)$ & $73.9(99 / 134)$ \\
\hline
\end{tabular}

AUC: area under the curve; axSpA: axial spondyloarthritis; BASMI: Bath Ankylosing Spondylitis Metrology Index; ES: Epionics SPINE; LCL: lower confidence limit; ROC: receiver-operating characteristic curve; RoK: range of kinematics; RoM: range of motion; Sens: sensitivity; Spec: specificity; UCL: upper confidence limit. 
Discrimination between r-axSpA $(A S)$ and $n r$-axSpA. The data showed that the ES variables of RoM flexion, extension, rotation, and lateral lumbar flexion are significantly different between $\mathrm{r}$-axSpA and nr-axSpA (covariable-adjusted results shown in the first column of the heatmap in Supplementary Figure 4 and unadjusted results are given in Supplementary Table 5, available with the online version of this article).

Covariable-adjusted ES variables as well as standardized questionnaires were assessed for their discriminative power between r-axSpA and nr-axSpA. Comparable results of AUC and sensitivity for a fixed minimal specificity of at least $80 \%$ are given in Table 3. The best individual result of $71.6 \%$ sensitivity was achieved for "range of motion in rotation" (RoM Rot). For the comprehensive model to distinguish between axSpA subgroups (details given in Supplementary Table 6, available with the online version of this article) and for the enlarged comprehensive model including BASFI (details given in Supplementary Table 7), a sensitivity of $70.1 \%$ of $80.5 \%$ are yielded, respectively.

The results of pairwise model comparisons to distinguish between patients with $\mathrm{r}$-axSpA and $\mathrm{nr}-\mathrm{axSpA}$ are presented in Table 3 (summarized in Supplementary Table 8, available with the online version of this article). It could be shown that the covariable-adjusted model of the ES variable RoM Rot yields significantly more true positives than the BASFI (for a given specificity of a minimum $\geq 80 \%, P=0.007$ ). Supporting raw ES variables (comprehensive model) did not further improve this result, but including the BASFI in the comprehensive model yielded systematically more true positives for a fixed specificity of a minimum $\geq 80 \%(P=0.002)$. Those ROC curves (analyzed in Supplementary Table 8) are visualized in Figure 3. There was a clear improvement relative to BASFI in discrimination between $\mathrm{r}$-axSpA and nr-axSpA as a result of the use of models based on combined and raw ES variables. However, the results for simple and more sophisticated models were quite similar. The significant improvement of sensitivity by including BASFI is relatively obvious but only at approximately $80 \%$ specificity. The results for discriminating between $\mathrm{r}$-axSpA and $\mathrm{nr}$-axSpA were compared with BASMI data. Only the enlarged comprehensive model including BASFI can show a significant improvement compared to BASMI $(P=0.005$, Figure 3$)$. Although the other smaller models and the single ES variable for rotation (RoM Rot) performed slightly better than the BASMI, there was no statistical significance (Supplementary Table 8).

\section{DISCUSSION}

The ES is an innovative tool to assess the spinal mobility of patients with axSpA. It shows promise to improve the objective quantification of spinal mobility in clinical studies and daily practice. In addition, the novel information on RoM and the kinematics of the back movement with the new aspects of speed and rotation are precisely measured.

In earlier studies in patients with mechanical back pain and HCs, ES sensors had already been shown to measure accurately with excellent test-retest reliability with evidence that the ES is a reliable measure of spinal mobility. $15,16,24,25,26$

Table 3. Results of ROC curves for modeling r-axSpA of covariable-adjusted models of single-derived ES variables, standardized questionnaires, the comprehensive model, and the enlarged comprehensive model including BASFI.

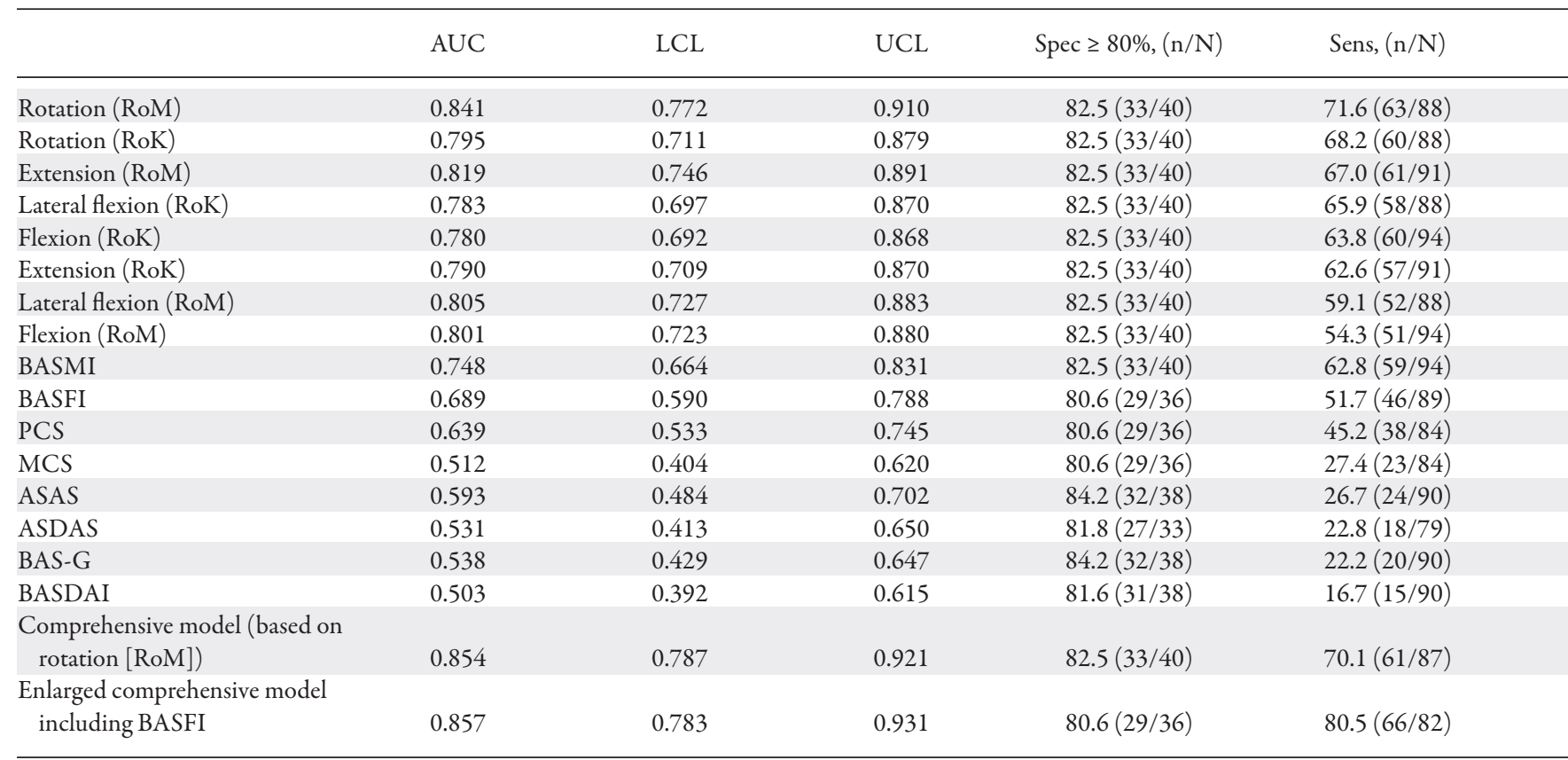

ASDAS: Ankylosing Spondylitis Disease Activity Score; ASAS: Assessment of Spondyloarthritis international Society; AUC: area under the curve; BASDAI Bath Ankylosing Spondylitis Disease Activity Score; BASFI: Bath Ankylosing Spondylitis Functional Index; BAS-G: Bath Patient Global Score; BASMI; Bath Ankylosing Spondylitis Metrology Index; ES: Epionics SPINE; LCL: lower confidence limit; MCS: mental component summary score of 12-item Short Form Health Survey; PCS: physical component summary score of 12-item Short Form Health Survey; ROC: receiver-operating curve; RoK: range of kinematics; RoM: range of motion; r-axSpA: radiographic axial spondyloarthritis; Sens: sensitivity; Spec: specificity; UCL: upper confidence limit. 

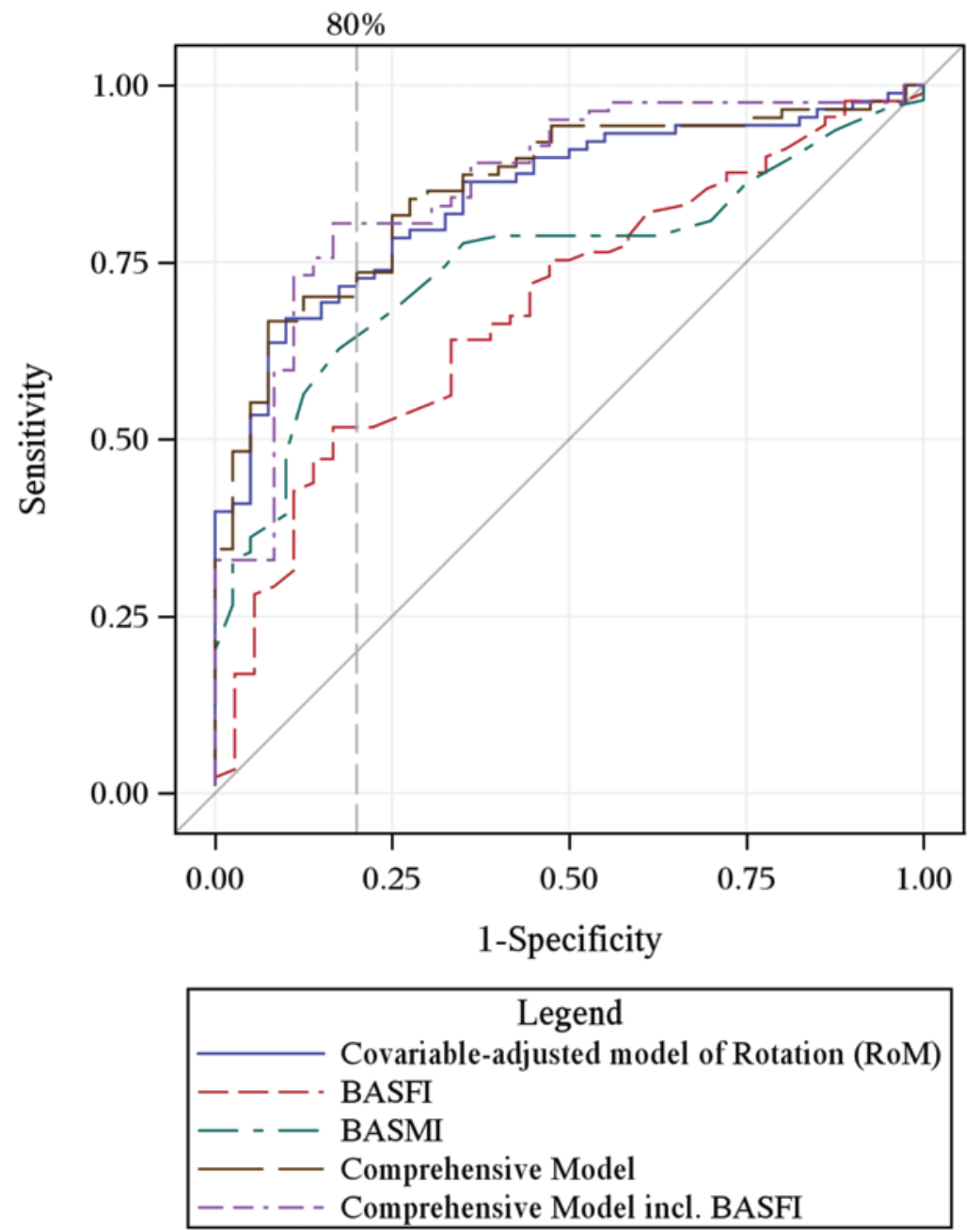

Figure 3. ROC curves for modeling r-axSpA of the covariable-adjusted model of the single Epionics SPINE variable RoM in rotation, BASFI only, BASMI only, the comprehensive model, and the enlarged comprehensive model including BASFI (missing data are excluded casewise). BASFI: Bath Ankylosing Spondylitis Functional Index; BASMI: Bath Ankylosing Spondylitis Metrology Index; r-axSpA: radiographic axial spondyloarthritis; ROC: receiver-operating characteristic curve; RoM: range of motion.

The results of the present study demonstrate that ES scores provide an excellent differentiation between patients with axSpA and HCs. Moreover, the ES was positively evaluated according to the specific imaging aspects of the OMERACT criteria with the aspects of truth, discrimination, and feasibility. $23,27,28$

As shown in Figure 1 and Supplementary Figure 3 (available with the online version of this article), there was a good correlation between BASMI and ES results, suggesting that the device does indeed measure what it intends to assess. As a result of this study, the content validity of the ES measurements, used in patients with axSpA, can be confirmed by showing significant correlations of spinal mobility in patients with axSpA compared to the BASMI. Within the individual BASMI measurements, lateral lumbar flexion, lumbar flexion, and cervical rotation showed the best correlation with ES variables.

The construct validity of the ES was confirmed by demonstrating that the spinal mobility measurements with the ES are capable of assessing the spinal mobility of patients with axSpA in detail, without any evidence of systematic errors. Thus, the device does measure what it intends to assess and therefore, fulfills the truth aspect of the OMERACT criteria. Further, according to the OMERACT criteria of discrimination, we showed that ES variables do yield additional information in differentiating between patients with axSpA and $\mathrm{HCs}$ as well as between axSpA subgroups. This differentiation could be demonstrated by comparing the mean values of raw ES results as well as by modeling the 2 groups in multivariable logistic regression models, with the selection of variables extended by factor analyses. Using BASFI scores in the enlarged comprehensive model, the best results for discrimination between $\mathrm{r}$-axSpA and $\mathrm{nr}-\mathrm{axSpA}$ in terms of sensitivity and specificity were reached. In addition, for the differentiation between r-axSpA and nr-axSpA, both the single ES variable rotation (RoM) and the 2 models demonstrated advantages compared to BASMI, although only 
for the enlarged comprehensive model was significance proven. Nevertheless, the use of these comprehensive models is explorative and not yet suitable for daily clinical use.

The OMERACT aspect of feasibility of the device is met because the tool is well standardized, simple to use, and the time to perform the examination does not exceed 15 minutes. The individually performed ES measurements are automated and computer guided. This guarantees accurate measures and intra- or interobserver variability is obviously not a concern. The presented results demonstrate that the specific aspects of the OMERACT criteria with the aspects of truth, discrimination, and feasibility are fulfilled by the ES. The BASFI results correlated only weakly with $\mathrm{ES}$ variables. This was expected because it has been described in previous observations that BASFI results can only partly assess and reflect patients' "real" mobility. 19,20

The study results also show that RoK and RoM scores decrease with increasing age. This expected finding was similarly observed in both groups, axSpA and HCs. Nevertheless, the exact measurement of spinal mobility in different planes using the ES seems to improve the assessment of function and mobility in patients with axSpA compared to the use of mere standardized questionnaires.

Further studies to demonstrate the sensitivity to change of therapies are ongoing, and there will be more coming in the future. Thereafter, the ES can be used to assess the mobility of patients in clinical studies with different interventions and also in daily practice.

Once validated in the field of axSpA, the ES may also be helpful in daily routine, as individual limitations in spinal mobility are directly visualized, thus enabling possible therapy approaches and helping to better understand and assess limitations in functional mobility and exact impairments of patients with axSpA.

Individual limitations measured with the ES will be directly presented to the patients with the help of a spider diagram that shows individual results and limitations in different colors (green: no limitations, yellow: moderate limitations, and red: major limitations). These limitations can be addressed by healthcare providers.

This approach, especially the use of the comprehensive models and the enlarged comprehensive model (including BASFI), is strongly explorative and to be validated in each step. It is not yet validated for the use in daily clinical practice.

We attempted to verify our results, for example, by using ranks instead of real variable values in factor analyses, and results did not notably differ. Nevertheless, the robustness of models is not ensured in this first explorative approach.

A further limitation is that there are missing data in several ES variables, which are not imputed (variables with $>10 \%$ missing data are not included in the analyses), and presented analyses were driven at complete cases, each. Thus, the number of patients included in pairwise comparisons and single models or estimations may differ. Further, restriction to complete cases might misleadingly improve diagnostic results. Because of the relatively small numbers, we did not compare nr-axSpA and HCs; however, no major differences are to be expected.
Finally, we did not assess the presence and extent of structural changes in the spine of patients with axSpA in this study. This will be an interesting research project in the future.

\section{ACKNOWLEDGMENT}

We acknowledge Dr. Uta Kiltz, MD, for her guidance in interpreting the results of this work.

\section{ONLINE SUPPLEMENT}

Supplementary material accompanies the online version of this article.

\section{REFERENCES}

1. Sieper J, van der Heijde D, Landewé R, Brandt J, Burgos-Vagas R, Collantes-Estevez E, et al. New criteria for inflammatory back pain in patients with chronic back pain: a real patient exercise by experts from the Assessment of SpondyloArthritis international Society (ASAS). Ann Rheum Dis 2009;68:784-8.

2. Rudwaleit M, van der Heijde D, Landewé R, Listing J, Akkoc N, Brandt J, et al. The development of Assessment of SpondyloArthritis international Society classification criteria for axial spondyloarthritis (part II): validation and final selection. Ann Rheum Dis 2009;68:777-83.

3. Kiltz U, Baraliakos X, Regel A, Buhring B, Braun J. Causes of pain in patients with axial spondyloarthritis. Clin Exp Rheumatol 2017;35 Suppl 107:102-7.

4. Kiltz U, van der Heijde D, Boonen A, Cieza A, Stucki G, Khan MA, et al. Development of a health index in patients with ankylosing spondylitis (ASAS HI): final result of a global initiative based on the ICF guided by ASAS. Ann Rheum Dis 2015;74:830-5.

5. Kiltz U, van der Heijde D, Boonen A, Akkoc N, Bautista-Molano W, Burgos-Vargas R, et al. Measurement properties of the ASAS Health Index: results of a global study in patients with axial and peripheral spondyloarthritis. Ann Rheum Dis 2018;77:1311-7.

6. Zochling J. Measures of symptoms and disease status in ankylosing spondylitis: Ankylosing Spondylitis Disease Activity Score (ASDAS), Ankylosing Spondylitis Quality of Life Scale (ASQoL), Bath Ankylosing Spondylitis Disease Activity Index (BASDAI), Bath Ankylosing Spondylitis Functional Index (BASFI), Bath Ankylosing Spondylitis Global Score (BAS-G), Bath Ankylosing Spondylitis Metrology Index (BASMI), Dougados Functional Index (DFI), and Health Assessment Questionnaire for the Spondylarthropathies (HAQ-S). Arthritis Care Res 2011;63 Suppl 11:S47-58

7. Zochling J, Braun J, van der Heijde D. Assessments in ankylosing spondylitis. Best Pract Res Clin Rheumatol 2006;20:521-37.

8. Braun J, Kiltz U, Sarholz M, Heldmann F, Regel A, Baraliakos X. Monitoring ankylosing spondylitis: clinically useful markers and prediction of clinical outcomes. Expert Rev Clin Immunol 2015;11:935-46.

9. van der Heijde DM, Revicki DA, Gooch KL, Wong RL, Kupper $\mathrm{H}$, Harnam N, et al. Physical function, disease activity, and health-related quality-of-life outcomes after 3 years of adalimumab treatment in patients with ankylosing spondylitis. Arthritis Res Ther 2009;11:R124.

10. van der Heijde D, Deodhar A, Inman RD, Braun J, Hsu B, Mack M. Comparison of three methods for calculating the Bath Ankylosing Spondylitis Metrology Index in a randomized placebo-controlled study. Arthritis Care Res 2012;64:1919-22.

11. Creemers MC, Franssen MJ, van't Hof MA, Gribnau FW, van de Putte LB, van Riel PL. Assessment of outcome in ankylosing spondylitis: an extended radiographic scoring system. Ann Rheum Dis 2005;64:127-9. 
12. Landewé $\mathrm{R}$, Dougados $\mathrm{M}$, Mielants $\mathrm{H}$, van der Tempel $\mathrm{H}$, van der Heijde D. Physical function in ankylosing spondylitis is independently determined by both disease activity and radiographic damage of the spine. Ann Rheum Dis 2009;68:863-7.

13. van Weely SF, Dekker J, Steultjens MP, van Denderen JC, Nurmohamed MT, Dijkmans BA, et al. Objective evaluation of physical functioning after tumor necrosis factor inhibitory therapy in patients with ankylosing spondylitis: a selection of 3 feasible performance-based tests. J Rheumatol 2015;42:623-9.

14. Consmüller T, Rohlmann A, Weinland D, Druschel C, Duda GN, Taylor WR. Comparative evaluation of a novel measurement tool to assess lumbar spine posture and range of motion. Eur Spine J 2012;21:2170-80.

15. Dideriksen JL, Gizzi L, Petzke F, Falla D. Deterministic accessory spinal movement in functional tasks characterizes individuals with low back pain. Clin Neurophysiol 2014;125:1663-8.

16. Vaisy M, Gizzi L, Petzke F, Consmüller T, Pfingsten M, Falla D. Measurement of lumbar spine functional movement in low back pain. Clin J Pain 2015;31:876-85.

17. Kiefer D, Baraliakos X, Bühring B, Kiltz U, Braun J. [Epionics SPINE-use of an objective method to examine spinal mobility in patients with axial spondyloarthritis]. [Article in German] Z Rheumatol 2019;78:773-4. Erratum in: Z Rheumatol 2020; 79:143-52.

18. Taylor WR, Consmüller T, Rohlmann A. A novel system for the dynamic assessment of back shape. Med Eng Phys 2010;32:1080-3.

19. van Weely SF, van Denderen JC, Steultjens MP, van der Leeden M, Nurmohamed MT, Dekker J, et al. Moving instead of asking? Performance-based tests and BASFI-questionnaire measure different aspects of physical function in ankylosing spondylitis. Arthritis Res Ther 2012;14:R52.

20. van Weely SF, van Denderen CJ, van der Horst-Bruinsma IE, Nurmohamed MT, Dijkmans BA, Dekker J, et al. Reproducibility of performance measures of physical function based on the BASFI, in ankylosing spondylitis. Rheumatology 2009;48:1254-60.
21. van Weely SF, van Denderen JC, Steultjens MP, Nurmohamed MT, Dijkmans BA, Dekker J, et al. What do we miss? ASAS non-responders on anti-TNF therapy show improvement in performance-based physical function. Rheumatology 2013; 52:1884-9.

22. Marques ML, Ramiro S, Goupille P, Dougados M, van Gaalen F, van der Heijde D. Measuring spinal mobility in early axial spondyloarthritis: does it matter? Rheumatology 2019; 58:1597-606

23. D’Agostino MA, Boers M, Kirwan J, van der Heijde D, Østergaard M, Schett G, et al. Updating the OMERACT filter: implications for imaging and soluble biomarkers. J Rheumatol 2014;41:1016-24.

24. Consmüller T, Rohlmann A, Weinland D, Druschel C, Duda GN, Taylor WR. Velocity of lordosis angle during spinal flexion and extension. PLoS One 2012;7:e50135.

25. Schmidt H, Bashkuev M, Weerts J, Altenscheidt J, Maier C, Reitmaier S. What does the shape of our back tell us? Correlation between sacrum orientation and lumbar lordosis. Spine J 2018;18:655-62.

26. Schmidt H, Bashkuev M, Weerts J, Graichen F, Altenscheidt J, Maier $\mathrm{C}$, et al. How do we stand? Variations during repeated standing phases of asymptomatic subjects and low back pain patients. J Biomech 2018;70:67-76.

27. Boers M, Brooks P, Strand CV, Tugwell P. The OMERACT filter for outcome measures in rheumatology. J Rheumatol 1998; 25:198-9.

28. Tugwell P, Boers M, D’Agostino MA, Beaton D, Boonen A, Bingham CO III, et al. Updating the OMERACT filter: implications of filter 2.0 to select outcome instruments through assessment of "truth": content, face, and construct validity. J Rheumatol 2014;41:1000-4. 